Supporting Information:

\title{
Layer-by-Layer Solution-Processed Organic Solar Cells with Perylene Diimides as Acceptors
}

Ming Hu, ${ }^{\text {at }}$ Youdi Zhang, ${ }^{\mathrm{a}, \mathrm{b}}$ Xia liu,${ }^{\mathrm{a}}{ }^{\ddagger}$ Xiaohong Zhao, ${ }^{\mathrm{a} *}$ Yu Hu, ${ }^{\mathrm{a}}$ Zhenyu Yang, ${ }^{\mathrm{a}}$ Changduk Yang, ${ }^{\mathrm{c}}$ Zhongyi Yuan, ${ }^{\mathrm{a}, \mathrm{b} *}$ and Yiwang Chen $^{\mathrm{a}, \mathrm{b}}$

${ }^{a}$ College of Chemistry, Nanchang University, 999 Xuefu Avenue, Nanchang 330031, China.

${ }^{b}$ Institute of Polymers and Energy Chemistry, Nanchang University, 999 Xuefu Avenue, Nanchang 330031, China.

${ }^{c}$ Department of Energy Engineering, School of Energy and Chemical Engineering, Perovtronics Research Center, Low Dimensional Carbon Materials Center, Ulsan National Institute of Science and Technology (UNIST), 50 UNIST-gil, Ulju-gun, Ulsan 44919, South Korea.

Author Contributions: M. Hu, Y. Zhang, and X. Liu contributed equally to this work.

Corresponding Author.

E-mail: zhaoxh@ncu.edu.cn (X. Zhao); yuan@ncu.edu.cn (Z. Yuan); yang@unist.ac.kr (C. Yang). 


\section{Table of Contents}

1. Supplementary Tables.

Table S1. Absorption and electrochemical data of TBDPDI-C T $_{5}$ TBDPDI- $\mathrm{C}_{11}$, and SdiPDI......S4

Table S2. Photovoltaic parameters of TBDPDI-C 5 -based LBL devices with different donors spincoated at $1500 \mathrm{rpm}$ for both donor and acceptor layer with inverted device architecture.

Table S3. Photovoltaic parameters of the OSCs based on TBDPDI-C 5 LBL films with different coating speeds of acceptor materials.

Table S4. Photovoltaic parameters of the OSCs based on TBDPDI-C 5 LBL films with different coating speeds of donor materials. S4

Table S5. Photovoltaic parameters of the OSCs based on TBDPDI-C 5 LBL films with different coating speeds of donor materials. .S5

Table S6. Photovoltaic parameters of the OSCs based on TBDPDI-C 5 LBL films with different coating speeds of donor materials. . $\mathrm{S} 5$

Table S7. Photovoltaic parameters of the LBL OSCs films spin-coated by $1000 \mathrm{rpm}$ for J60 and $1500 \mathrm{rpm}$ for TBDPDI-C 5 with different annealing temperatures. S5

Table S8. Photovoltaic parameters of BHJ and LBL OSCs with different device structures......S6

Table S9. Hole and electron mobility of the three acceptors-based BHJ and LBL devices. .......S6 Table S10. In-plane GIWAXS data of J60, TBDPDI-C 5 , TBDPDI-C 11 , and SdiPDI neat films, BHJ, and LBL films.

Table S11. Out-of-plane GIWAXS data of J60, TBDPDI-C 5 , TBDPDI-C 11 , and SdiPDI neat films, BHJ, and LBL films. S7

Table S12. Contact angle and surface energy of water and glycerol measured by Wu model. ...S8

2. Supplementary Figures . S8

Figure S1. Chemical structures of five different donor polymers.... . $\mathrm{S} 8$

Figure S2. UV-vis absorption spectra of (a) TBDPDI-C $5^{-}$, (b) TBDPDI-C $1^{-}$, and (c) SdiPDIbased LBL BHJ films S8

Figure S3. $J$ - $V$ curves of (a) TBDPDI-C $11^{-}$(b) SdiPDI- based LBL and BHJ devices. .S9

Figure S4. EQE spectra and integrated $J_{\mathrm{sc}}$ of (a) TBDPDI-C ${ }_{11}$ (b) SdiPDI based LBL and BHJ devices .S9 
Figure S5. (a-b) Photocurrent density $\left(J_{\mathrm{ph}}\right)$ plotted against the effective voltage $\left(V_{\text {eff }}\right)$ spectra, light intensity dependence of (c-d) $J_{\text {sc }}$, and (e-f) $V_{\text {oc }}$ for TBDPDI-C 11 and SdiPDI based LBL and BHJ devices S10

Figure S6. Current density versus voltage characteristics of (a-b) TBDPDI-C $\mathrm{C}_{11}$ and (c-d) SdiPDI based LBL and BHJ hole-only and electron-only devices. $\mathrm{S} 10$ Figure S7. AFM height (a-f) and phase (g-l) images of TBDPDI-C $\mathrm{C}_{11}$ and SdiPDI pristine, LBL, and BHJ films. . $\mathrm{S} 10$

Figure S8. 2D GIWAXS diffraction patterns of (a) neat TBDPDI-C $\mathrm{C}_{11}$, (b) neat SdiPDI, (c) TBDPDI-C 11 LBL, (d) TBDPDI-C 11 BHJ, (e) SdiPDI LBL, and (f) SdiPDI BHJ films. (g) Corresponding 1D line-cuts of 2D GIWAX patterns.

Figure S9. Contact angles of water and glycerol on PEDOT:PSS, ZnO, J60, TBDPDI-C 5 , LBL film, and BHJ film. S11 


\section{Supplementary Tables}

Table S1. Absorption and electrochemical data of TBDPDI-C 5 , TBDPDI-C 11 , and SdiPDI.

\begin{tabular}{ccccc}
\hline Materials & $\begin{array}{c}\lambda_{\text {max }^{\mathbf{a}}} \\
(\mathrm{nm})\end{array}$ & $\begin{array}{c}\boldsymbol{E}_{\mathbf{g}}^{\mathbf{b}} \\
(\mathrm{eV})\end{array}$ & $\begin{array}{c}\text { LUMO } \\
(\mathrm{eV})\end{array}$ & $\begin{array}{c}\text { HOMO }^{\mathbf{c}} \\
(\mathrm{eV})\end{array}$ \\
\hline TBDPDI-C $_{5}$ & 480 & 2.06 & -3.73 & -5.79 \\
TBDPDI-C $_{11}$ & 480 & 2.07 & -3.75 & -5.82 \\
SdiPDI & 534 & 2.09 & -4.04 & -6.13 \\
\hline
\end{tabular}

${ }^{\mathrm{a}}$ Measured in solid statue; ${ }^{\mathrm{b}}$ Optical bandgap; ${ }^{\mathrm{c}} \mathrm{HOMO}=\mathrm{LUMO}-E_{\mathrm{g}}$.

Table S2. Photovoltaic parameters of TBDPDI-C 5 -based LBL devices with different donors spin-coated at $1500 \mathrm{rpm}$ for both donor and acceptor layer with inverted device architecture.

\begin{tabular}{ccccc}
\hline Donors & $\begin{array}{c}\boldsymbol{V}_{\mathbf{~ o c}} \\
{[\mathrm{V}]}\end{array}$ & $\begin{array}{c}\boldsymbol{J}_{\text {sc }} \\
{\left[\mathrm{mA} \mathrm{cm}^{-2}\right]}\end{array}$ & $\begin{array}{c}\text { FF } \\
{[\%]}\end{array}$ & $\begin{array}{c}\text { PCE } \\
{[\%]}\end{array}$ \\
\hline PTB7-Th & 0.955 & 11.93 & 45.73 & 5.23 \\
PBDB-T & 0.994 & 9.36 & 57.87 & 5.41 \\
PDBD-T1 & 0.925 & 8.32 & 39.52 & 3.05 \\
PBDB-T-2F & 1.064 & 6.84 & 53.53 & 3.91 \\
J60 & 0.968 & 9.42 & 63.09 & 5.77 \\
\hline
\end{tabular}

All devices were measured under the illumination of $\mathrm{AM} 1.5 \mathrm{G}, 100 \mathrm{~mW} \mathrm{~cm}^{-2}$

Table S3. Photovoltaic parameters of the OSCs based on TBDPDI-C 5 LBL films with different coating speeds of acceptor materials.

\begin{tabular}{ccccc}
\hline $\begin{array}{c}\text { Speeding }(\mathbf{r p m}) \\
\left(\mathrm{J} 60 / \mathrm{TBDPDI}-\mathrm{C}_{5}\right)\end{array}$ & $\begin{array}{c}\boldsymbol{V}_{\mathbf{~ o c}} \\
{[\mathrm{V}]}\end{array}$ & $\begin{array}{c}\boldsymbol{J}_{\mathbf{s c}} \\
{\left[\mathrm{mA} \mathrm{cm}^{-2}\right]}\end{array}$ & $\begin{array}{c}\text { FF } \\
{[\%]}\end{array}$ & $\begin{array}{c}\text { PCE } \\
{[\%]}\end{array}$ \\
\hline $1000 / 1000$ & 0.964 & 10.76 & 55.39 & 5.75 \\
$1000 / 1300$ & 0.972 & 11.05 & 56.16 & 6.03 \\
$1000 / 1500$ & 0.969 & 10.57 & 59.62 & 6.11 \\
$1000 / 1800$ & 0.968 & 10.40 & 59.24 & 5.97 \\
\hline
\end{tabular}

All devices were measured under the illumination of AM $1.5 \mathrm{G}, 100 \mathrm{~mW} \mathrm{~cm}{ }^{-2}$

Table S4. Photovoltaic parameters of the OSCs based on TBDPDI-C 5 LBL films with different coating speeds of donor materials.

\begin{tabular}{ccccc}
\hline $\begin{array}{c}\text { Speeding (rpm) } \\
\left(\mathrm{J} 60 / \mathrm{TBDPDI}-\mathrm{C}_{5}\right)\end{array}$ & $\begin{array}{c}\boldsymbol{V}_{\mathbf{~ o c}} \\
{[\mathrm{V}]}\end{array}$ & $\begin{array}{c}\boldsymbol{J}_{\mathbf{s c}} \\
{\left[\mathrm{mA} \mathrm{cm}^{-2}\right]}\end{array}$ & $\begin{array}{c}\text { FF } \\
{[\%]}\end{array}$ & $\begin{array}{c}\text { PCE } \\
{[\%]}\end{array}$ \\
\hline $1300 / 1000$ & 0.968 & 10.08 & 58.74 & 5.74 \\
$1300 / 1300$ & 0.970 & 10.24 & 58.70 & 5.83 \\
$1300 / 1500$ & 0.966 & 11.04 & 55.23 & 5.89 \\
$1300 / 1800$ & 0.960 & 10.76 & 52.98 & 5.47 \\
\hline
\end{tabular}

All devices were measured under the illumination of AM 1.5G, $100 \mathrm{~mW} \mathrm{~cm}^{-2}$ 
Table S5. Photovoltaic parameters of the OSCs based on TBDPDI-C 5 LBL films with different coating speeds of donor materials.

\begin{tabular}{ccccc}
\hline $\begin{array}{c}\text { Speeding }(\mathbf{r p m}) \\
\left(\mathrm{J} 60 / \mathrm{TBDPDI}-\mathrm{C}_{5}\right)\end{array}$ & $\begin{array}{c}\boldsymbol{V}_{\mathbf{o c}} \\
{[\mathrm{V}]}\end{array}$ & $\begin{array}{c}\boldsymbol{J}_{\mathbf{s c}} \\
{\left[\mathrm{mA} \mathrm{cm}^{-2}\right]}\end{array}$ & $\begin{array}{c}\text { FF } \\
{[\%]}\end{array}$ & $\begin{array}{c}\text { PCE } \\
{[\%]}\end{array}$ \\
\hline $1500 / 1000$ & 0.958 & 9.73 & 57.38 & 5.35 \\
$1500 / 1300$ & 0.963 & 9.70 & 61.19 & 5.71 \\
$1500 / 1500$ & 0.958 & 9.80 & 58.86 & 5.53 \\
$1500 / 1800$ & 0.965 & 9.91 & 60.98 & 5.83 \\
\hline
\end{tabular}

All devices were measured under the illumination of AM $1.5 \mathrm{G}, 100 \mathrm{~mW} \mathrm{~cm}{ }^{-2}$

Table S6. Photovoltaic parameters of the OSCs based on TBDPDI-C 5 LBL films with different coating speeds of donor materials.

\begin{tabular}{ccccc}
\hline $\begin{array}{c}\text { Speeding (rpm) } \\
(\mathrm{J} 60 / \text { TBDPDI-C })\end{array}$ & $\boldsymbol{V}_{\mathbf{o c}}$ & $\boldsymbol{J}_{\text {sc }}$ & $\mathbf{F F}$ & PCE \\
\hline $1800 / 1000$ & {$[\mathrm{~V}]$} & {$\left[\mathrm{mA} \mathrm{cm}^{-2}\right]$} & {$[\%]$} & {$[\%]$} \\
$1800 / 1300$ & 0.955 & 9.60 & 58.06 & 5.33 \\
$1800 / 1500$ & 0.957 & 9.80 & 54.89 & 5.15 \\
$1800 / 1800$ & 0.965 & 9.45 & 59.84 & 5.45 \\
\hline
\end{tabular}

All devices were measured under the illumination of AM $1.5 \mathrm{G}, 100 \mathrm{~mW} \mathrm{~cm}^{-2}$

Table S7. Photovoltaic parameters of the LBL OSCs films spin-coated by $1000 \mathrm{rpm}$ for J60 and 1500 rpm for TBDPDI- $\mathrm{C}_{5}$ with different annealing temperatures.

\begin{tabular}{ccccc}
\hline TA & $\boldsymbol{V}_{\text {oc }}$ & $\boldsymbol{J}_{\text {sc }}$ & FF & PCE \\
{$\left[{ }^{\circ} \mathrm{C}\right]$} & {$[\mathrm{V}]$} & {$\left[\mathrm{mA} \mathrm{cm}^{-2}\right]$} & {$[\%]$} & {$[\%]$} \\
\hline w/o & 0.954 & 9.61 & 58.36 & 5.35 \\
80 & 0.957 & 9.92 & 60.43 & 5.74 \\
100 & 0.969 & 10.57 & 59.62 & 6.11 \\
120 & 0.956 & 9.81 & 60.51 & 5.66 \\
150 & 0.959 & 9.65 & 58.87 & 5.45 \\
\hline
\end{tabular}

All devices were measured under the illumination of AM $1.5 \mathrm{G}, 100 \mathrm{~mW} \mathrm{\textrm {cm } ^ { - 2 }}$ 
Table S8. Photovoltaic parameters of BHJ and LBL OSCs with different device structures.

\begin{tabular}{|c|c|c|c|c|c|}
\hline Active layer & $\begin{array}{c}\text { Device } \\
\text { Configuration } \\
\end{array}$ & $\begin{array}{r}V_{\text {oc }} \\
{[\mathrm{V}]} \\
\end{array}$ & $\begin{array}{c}\boldsymbol{J}_{\mathbf{s c}} \\
{\left[\mathrm{mA} \mathrm{cm}^{-2}\right]} \\
\end{array}$ & $\begin{array}{l}\text { FF } \\
{[\%]} \\
\end{array}$ & $\begin{array}{r}\text { PCE } \\
{[\%]} \\
\end{array}$ \\
\hline TBDPDI-C $_{5}$ & conventional & 0.943 & 9.89 & 54.77 & 5.11 \\
\hline$(\mathrm{BHJ})$ & inverted & 0.950 & 9.46 & 57.15 & 5.14 \\
\hline TBDPDI-C $_{5}$ & conventional & 0.947 & 11.08 & 54.38 & 5.71 \\
\hline (LBL) & inverted & 0.969 & 10.57 & 59.62 & 6.11 \\
\hline TBDPDI-C ${ }_{11}$ & conventional & 0.940 & 10.34 & 45.97 & 4.47 \\
\hline$(\mathrm{BHJ})$ & inverted & 0.942 & 9.34 & 54.78 & 4.82 \\
\hline TBDPDI-C $_{11}$ & conventional & 0.942 & 9.72 & 53.40 & 4.89 \\
\hline (LBL) & inverted & 0.946 & 9.67 & 54.70 & 5.00 \\
\hline SdiPDI & conventional & 0.738 & 9.02 & 39.14 & 2.61 \\
\hline$(\mathrm{BHJ})$ & inverted & 0.813 & 8.44 & 45.29 & 3.11 \\
\hline SdiPDI & conventional & 0.760 & 9.65 & 43.63 & 3.20 \\
\hline (LBL) & inverted & 0.823 & 8.51 & 51.42 & 3.60 \\
\hline
\end{tabular}

All devices were measured under the illumination of AM $1.5 \mathrm{G}, 100 \mathrm{~mW} \mathrm{\textrm {cm } ^ { - 2 }}$

Table S9. Hole and electron mobility of the three acceptors-based BHJ and LBL devices.

\begin{tabular}{|c|c|c|c|c|}
\hline Materials & Film structure & $\begin{array}{c}\boldsymbol{\mu}_{\mathbf{h}} \\
{\left[\mathrm{cm}^{2} \mathrm{~V}^{-1} \mathrm{~s}^{-1}\right]}\end{array}$ & $\begin{array}{c}\boldsymbol{\mu}_{\mathrm{e}} \\
{\left[\mathrm{cm}^{2} \mathrm{~V}^{-1} \mathrm{~s}^{-1}\right]}\end{array}$ & $\mu_{\mathrm{h}} / \mu_{\mathrm{e}}$ \\
\hline \multirow{2}{*}{$\mathrm{TBDPDI} \mathrm{C}_{5}$} & BHJ & $1.69 \times 10^{-4}$ & $0.52 \times 10^{-4}$ & 3.25 \\
\hline & $\mathrm{LbL}$ & $2.23 \times 10^{-4}$ & $1.48 \times 10^{-4}$ & 1.51 \\
\hline \multirow{2}{*}{ TBDPDI-C $_{11}$} & BHJ & $0.70 \times 10^{-4}$ & $0.46 \times 10^{-4}$ & 1.52 \\
\hline & $\mathrm{LbL}$ & $1.75 \times 10^{-4}$ & $0.82 \times 10^{-4}$ & 2.13 \\
\hline \multirow{2}{*}{ SdiPDI } & BHJ & $0.86 \times 10^{-4}$ & $0.12 \times 10^{-4}$ & 8.00 \\
\hline & $\mathrm{LbL}$ & $0.98 \times 10^{-4}$ & $0.73 \times 10^{-4}$ & 1.34 \\
\hline
\end{tabular}


Table S10. In-plane GIWAXS data of J60, TBDPDI-C , TBDPDI-C $_{11}$, and SdiPDI neat films, BHJ, and LBL films.

\begin{tabular}{|c|c|c|c|c|c|c|c|c|}
\hline In-plane & $\begin{array}{c}(100) \\
Q\end{array}$ & $\begin{array}{c}(\mathbf{1 0 0 )} \\
d \text {-spacing } \\
(\AA)\end{array}$ & $\begin{array}{c}\text { (100) } \\
\text { FWHM } \\
\Delta \theta\end{array}$ & $\begin{array}{c}\text { (100) } \\
\text { Crystal size } \\
(\AA)\end{array}$ & $\begin{array}{c}(010) \\
\mathbf{Q}\end{array}$ & $\begin{array}{c}\text { (010) } \\
d \text {-spacing } \\
(\AA)\end{array}$ & $\begin{array}{c}\text { (010) } \\
\text { FWHM } \\
\Delta \theta\end{array}$ & $\begin{array}{c}\text { (010) } \\
\text { Crystal size } \\
(\AA)\end{array}$ \\
\hline $\mathrm{J} 60$ & 0.27 & 23.10 & 0.11 & 51.42 & 1.77 & 3.54 & 0.17 & 34.70 \\
\hline TBDPDI-C $_{5}$ & 0.33 & 18.98 & 0.19 & 29.62 & 1.63 & 3.85 & 0.63 & 9.05 \\
\hline TBDPDI-C $_{11}$ & 0.34 & 18.70 & 0.13 & 43.19 & 1.53 & 4.05 & 0.75 & 7.63 \\
\hline SdiPDI & 0.35 & 17.95 & 0.17 & 32.70 & N/A & N/A & N/A & N/A \\
\hline TBDPDI-C $_{5}(\mathrm{BHJ})$ & 0.32 & 19.76 & 0.14 & 40.12 & 1.67 & 3.77 & 0.47 & 12.19 \\
\hline TBDPDI-C $_{5}(\mathrm{LBL})$ & 0.30 & 20.81 & 0.23 & 24.59 & 1.71 & 3.68 & 0.26 & 21.83 \\
\hline TBDPDI-C $_{11}(\mathrm{BHJ})$ & 0.30 & 21.23 & 0.08 & 67.34 & 1.67 & 3.76 & 0.39 & 14.73 \\
\hline TBDPDI-C $_{11}(\mathrm{LBL})$ & 0.30 & 20.74 & 0.09 & 64.28 & 1.64 & 3.84 & 0.45 & 12.67 \\
\hline SdiPDI (BHJ) & 0.29 & 21.74 & 0.08 & 75.42 & 1.67 & 3.77 & 0.36 & 15.70 \\
\hline SdiPDI (LBL) & 0.29 & 21.44 & 0.09 & 62.85 & 1.66 & 3.79 & 0.38 & 14.92 \\
\hline
\end{tabular}

Table S11. Out-of-plane GIWAXS data of J60, TBDPDI-C ${ }_{5}$, TBDPDI-C 11 , and SdiPDI neat films, BHJ, and LBL films.

\begin{tabular}{|c|c|c|c|c|c|c|c|c|}
\hline Out-of-plane & $\begin{array}{c}(\mathbf{1 0 0}) \\
\mathbf{Q}\end{array}$ & $\begin{array}{c}\text { (100) } \\
d \text {-spacing } \\
(\AA)\end{array}$ & $\begin{array}{c}\text { (100) } \\
\text { FWHM } \\
\Delta \theta\end{array}$ & $\begin{array}{c}\text { (100) } \\
\text { Crystal size } \\
(\AA)\end{array}$ & $\begin{array}{c}\text { (010) } \\
\mathbf{Q}\end{array}$ & $\begin{array}{c}\text { (010) } \\
d \text {-spacing } \\
(\AA)\end{array}$ & $\begin{array}{c}\text { (010) } \\
\text { FWHM } \\
\Delta \theta\end{array}$ & $\begin{array}{c}\text { (010) } \\
\text { Crystal size } \\
(\AA)\end{array}$ \\
\hline $\mathrm{J} 60$ & 0.26 & 24.54 & 0.11 & 50.06 & 1.70 & 3.69 & 0.20 & 28.31 \\
\hline TBDPDI-C $_{5}$ & N/A & N/A & N/A & N/A & 1.68 & 3.75 & 0.49 & 11.57 \\
\hline TBDPDI-C $_{11}$ & 0.28 & 22.85 & 0.24 & 23.77 & 1.62 & 3.87 & 0.40 & 14.43 \\
\hline SdiPDI & 0.27 & 23.27 & 0.21 & 26.56 & N/A & N/A & N/A & N/A \\
\hline $\mathrm{TBDPDI}^{-\mathrm{C}_{5}}(\mathrm{BHJ})$ & 0.19 & 32.73 & 0.27 & 20.72 & 1.72 & 3.66 & 0.25 & 22.88 \\
\hline $\mathrm{TBDPDI} \mathrm{C}_{5}(\mathrm{LbL})$ & N/A & N/A & N/A & N/A & 1.66 & 3.78 & 0.41 & 13.87 \\
\hline TBDPDI-C $_{11}(\mathrm{BHJ})$ & 0.19 & 32.56 & 0.26 & 21.67 & 1.72 & 3.66 & 0.22 & 26.36 \\
\hline TBDPDI-C $_{11}(\mathrm{LbL})$ & 0.17 & 36.96 & 0.35 & 16.11 & 1.72 & 3.66 & 0.22 & 26.12 \\
\hline SdiPDI (BHJ) & 0.24 & 26.29 & 0.19 & 29.15 & 1.72 & 3.66 & 0.18 & 31.78 \\
\hline SdiPDI (LbL) & 0.27 & 23.27 & 0.16 & 35.13 & 1.72 & 3.66 & 0.19 & 29.80 \\
\hline
\end{tabular}


Table S12. Contact angle and surface energy of water and glycerol measured by Wu model.

\begin{tabular}{|c|c|c|c|c|c|}
\hline \multirow{2}{*}{ Film } & \multicolumn{2}{|c|}{ Contact angle $\left[{ }^{\circ}\right]$} & \multirow{2}{*}{$\begin{array}{c}\boldsymbol{\gamma}_{\mathrm{s}}^{\mathbf{d}} \\
{\left[\mathrm{mN} \mathrm{m}^{-1}\right]}\end{array}$} & \multirow{2}{*}{$\begin{array}{c}\gamma_{\mathrm{s}}^{\mathbf{p}} \\
{\left[\mathrm{mN} \mathrm{m}^{-1}\right]}\end{array}$} & \multirow{2}{*}{$\begin{array}{c}\gamma_{\mathrm{s}} \\
{\left[\mathrm{mN} \mathrm{m}^{-1}\right]}\end{array}$} \\
\hline & Water & Glycerol & & & \\
\hline PEDOT:PSS & 19.88 & 42.25 & 30.00 & 5.00 & 35.00 \\
\hline $\mathrm{ZnO}$ & 45.66 & 40.70 & 19.91 & 34.32 & 54.23 \\
\hline J60 & 103.14 & 87.12 & 21.44 & 3.47 & 24.92 \\
\hline TBDPDI-C $_{5}$ & 98.37 & 90.21 & 11.58 & 9.47 & 21.05 \\
\hline PEDOT:PSS/J60/TBDPDI-C ${ }_{5}$ & 104.35 & 97.48 & 9.36 & 8.30 & 17.66 \\
\hline PEDOT:PSS/J60:TBDPDI-C & 104.45 & 95.25 & 11.99 & 6.70 & 18.69 \\
\hline $\mathrm{ZnO} / \mathrm{J} 60 / \mathrm{TBDPDI}-\mathrm{C}_{5}$ & 104.54 & 96.24 & 10.92 & 7.26 & 18.18 \\
\hline $\mathrm{ZnO} / \mathrm{J} 60: \mathrm{TBDPDI}-\mathrm{C}_{5}$ & 104.71 & 95.27 & 12.25 & 6.46 & 18.71 \\
\hline
\end{tabular}

\section{Supplementary Figures}
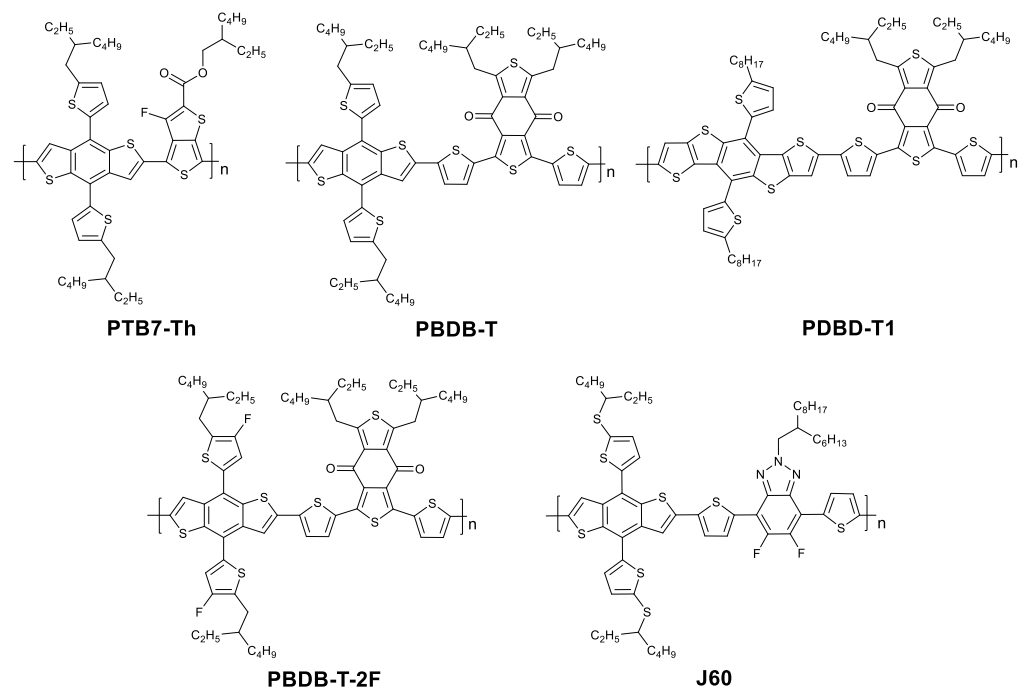

Figure S1. Chemical structures of five different donor polymers.
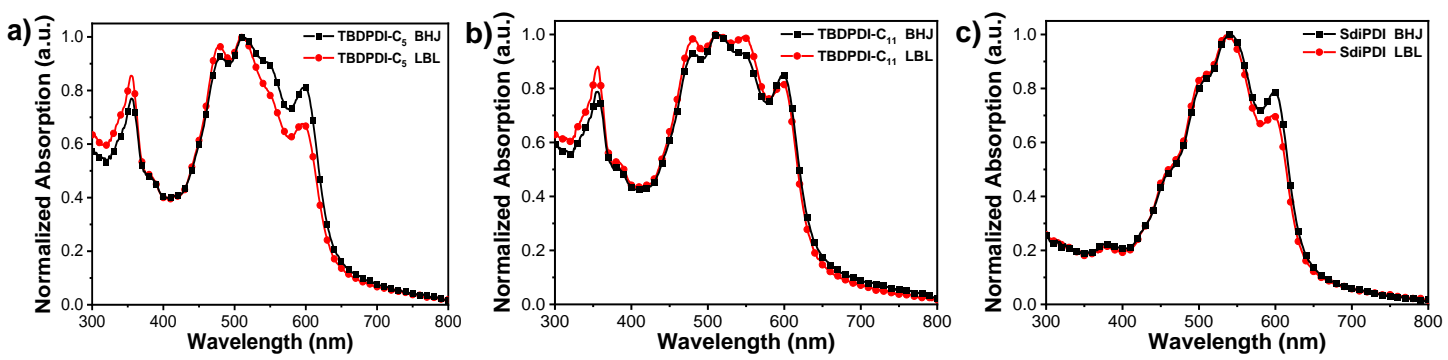

Figure S2. UV-vis absorption spectra of (a) TBDPDI-C5-, (b) TBDPDI-C11-, and (c) SdiPDI-based LBL BHJ films. 

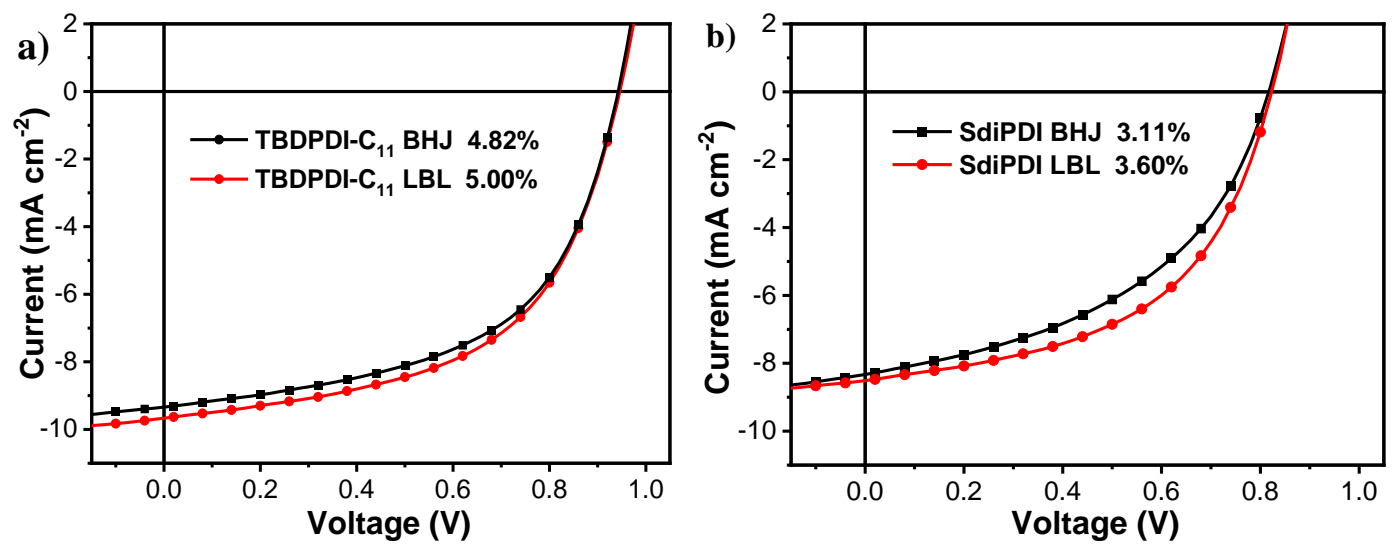

Figure S3. $J-V$ curves of (a) TBDPDI-C 11 - (b) SdiPDI- based LBL and BHJ devices.
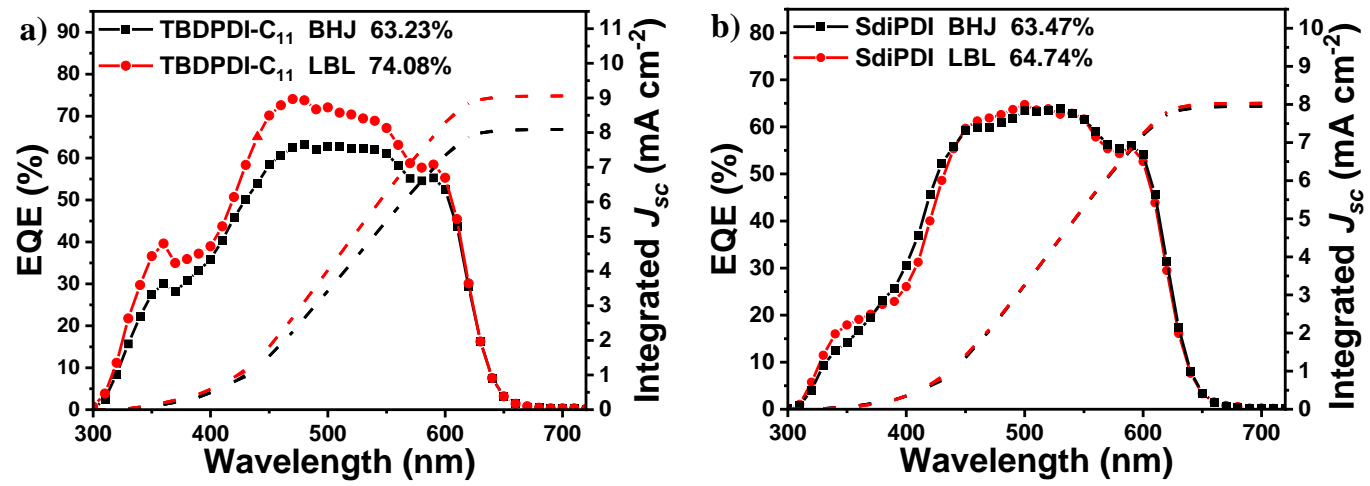

Figure S4. EQE spectra and integrated $J_{\text {sc }}$ of (a) TBDPDI-C 11 (b) SdiPDI based LBL and BHJ devices.
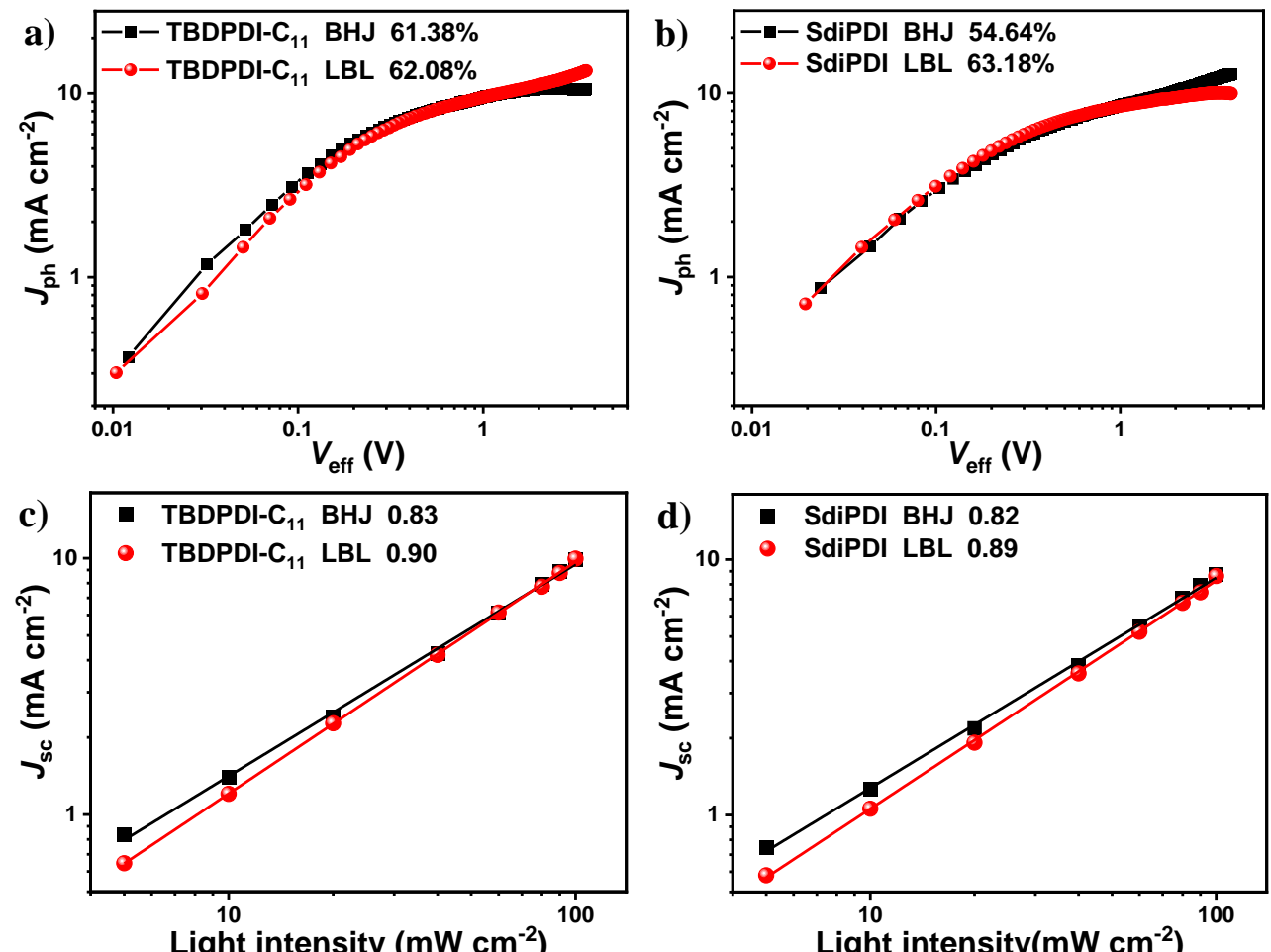

Light intensity $\left(\mathrm{mW} \mathrm{cm}^{-2}\right)$ 

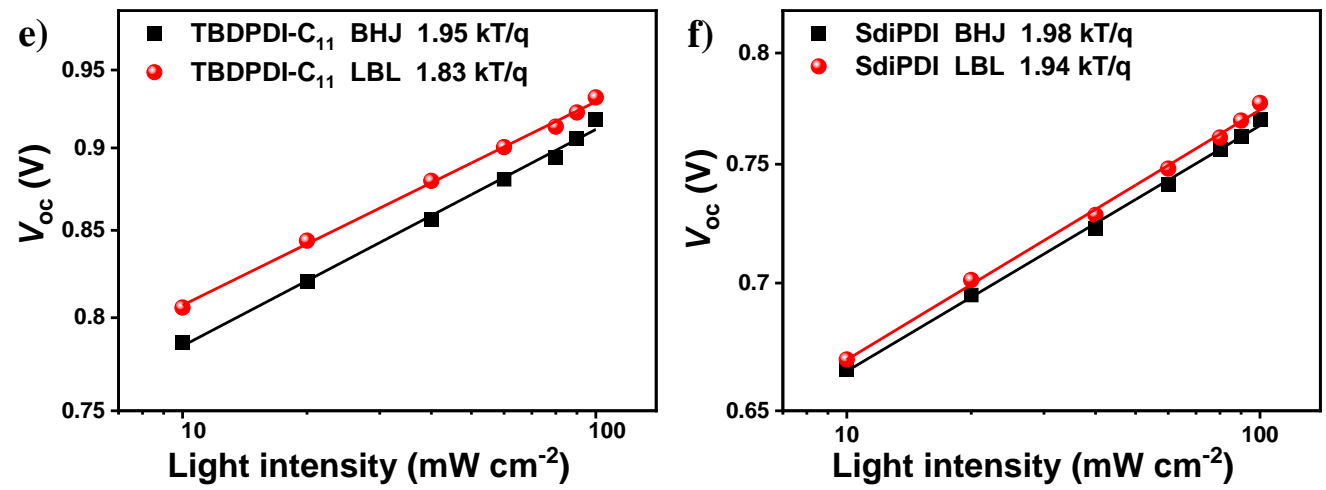

Figure S5. (a-b) Photocurrent density $\left(J_{\mathrm{ph}}\right)$ plotted against the effective voltage $\left(V_{\text {eff }}\right)$ spectra, light intensity dependence of (c-d) $J_{\mathrm{sc}}$, and (e-f) $V_{\text {oc }}$ for TBDPDI- $\mathrm{C}_{11}$ and SdiPDI based LBL and BHJ devices.
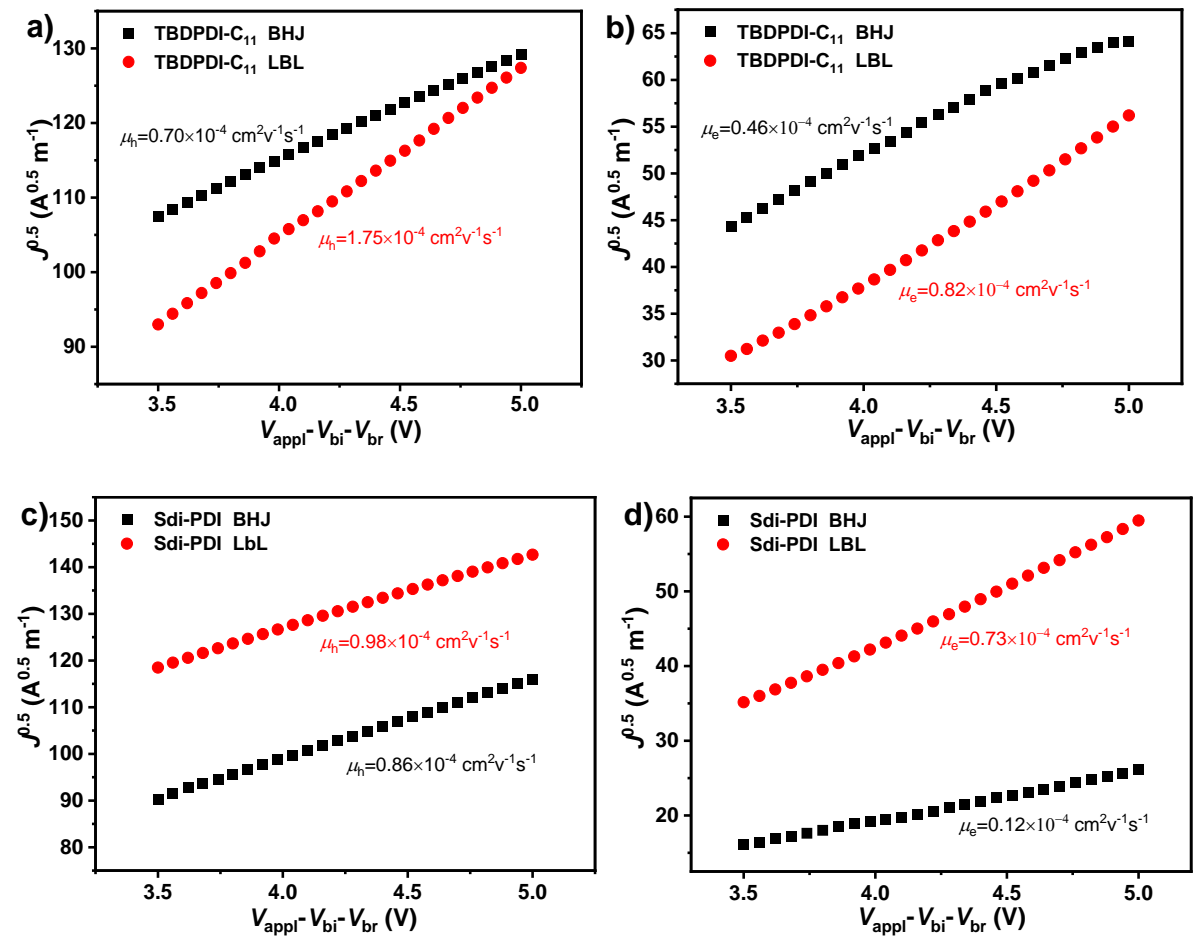

Figure S6. Current density versus voltage characteristics of (a-b) TBDPDI-C $\mathrm{C}_{11}$ and (c-d) SdiPDI based LBL and BHJ hole-only and electron-only devices.

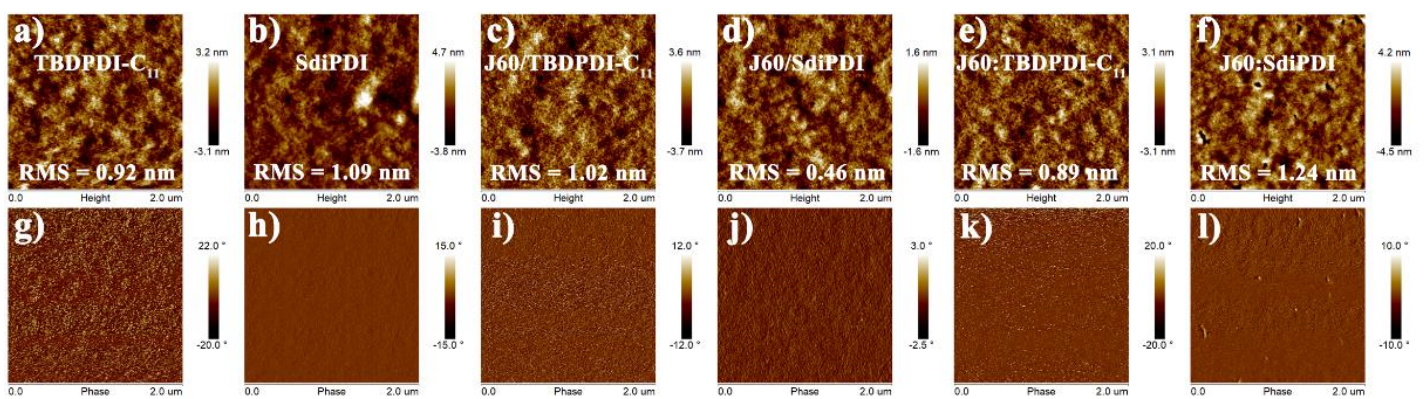

Figure S7. AFM height (a-f) and phase (g-l) images of TBDPDI- $\mathrm{C}_{11}$ and SdiPDI pristine, LBL, and BHJ films. 

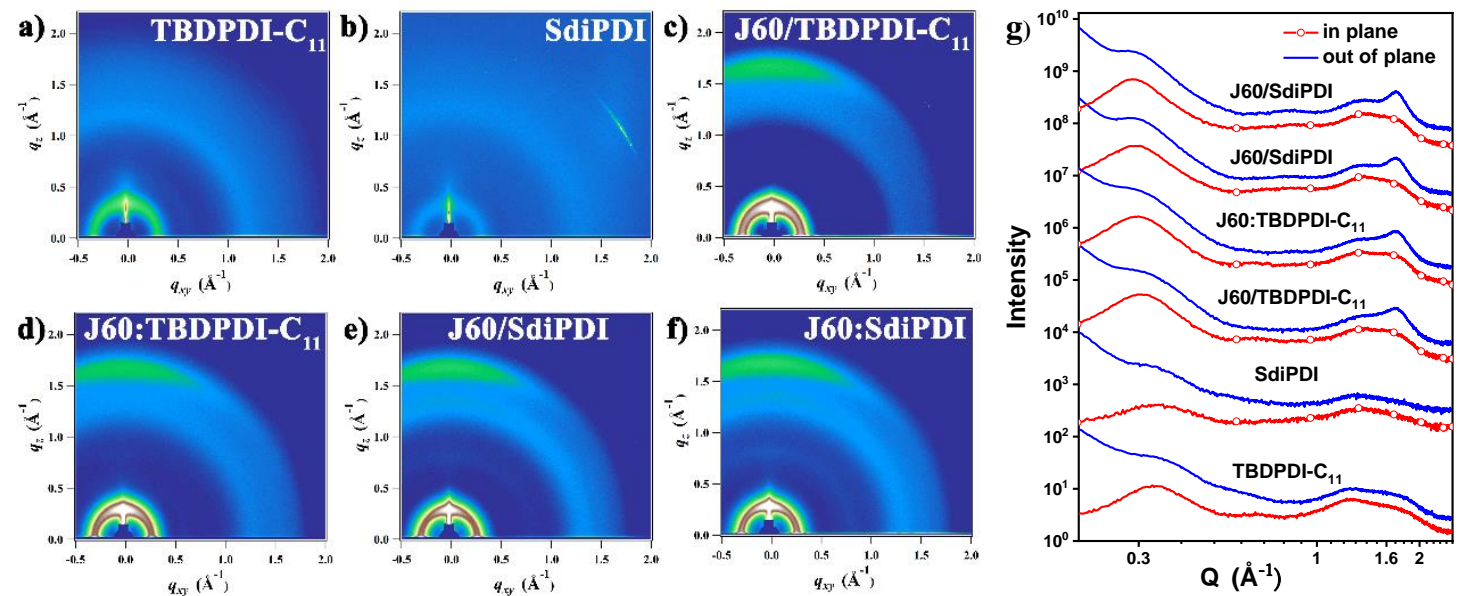

Figure S8. 2D GIWAXS diffraction patterns of (a) neat TBDPDI-C ${ }_{11}$, (b) neat SdiPDI, (c) TBDPDI-C ${ }_{11}$ LBL, (d) TBDPDI-C ${ }_{11}$ BHJ, (e) SdiPDI LBL, and (f) SdiPDI BHJ films. (g) Corresponding 1D line-cuts of 2D GIWAX patterns.

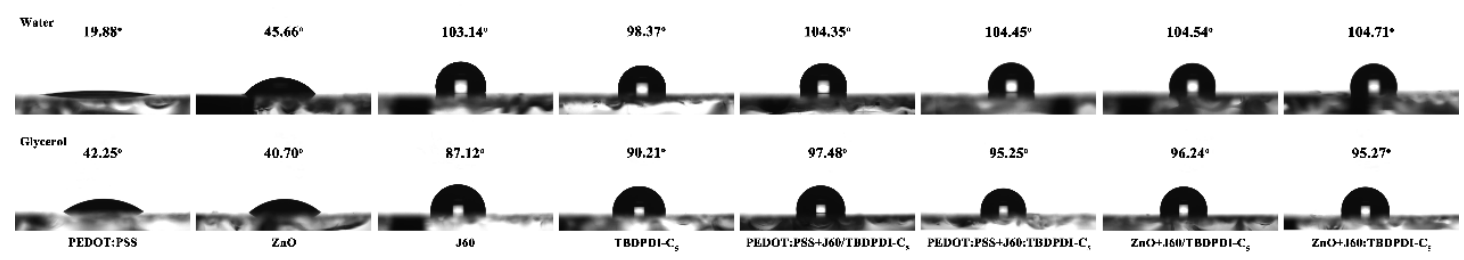

Figure S9. Contact angles of water and glycerol on PEDOT:PSS, ZnO, J60, TBDPDI-C ${ }_{5}$, LBL film, and BHJ film. 